\title{
Geometrically non-linear free and forced vibration of a shallow arch
}

\author{
Omar Outassafte ${ }^{1}$, Ahmed Adri' ${ }^{2}$, Yassine El Khouddar ${ }^{3}$, Said Rifai ${ }^{4}$, Rhali Benamar ${ }^{5}$ \\ ${ }_{1,2,3,4}$ Hassan II University of Casablanca, EST, LMPGI, B.P.8012, Oasis Casablanca, Morocco \\ ${ }^{5}$ Mohammed V University in Rabat, EMI-Rabat, LERSIM, Agdal, B.P. 765, Rabat, Morocco \\ ${ }^{5}$ Corresponding author \\ E-mail: ${ }^{1}$ omar.outassafte@ensem.ac.ma, ${ }^{2}$ ahmedadri@gmail.com, ${ }^{3}$ yassine.elkhouddar@ensem.ac.ma, \\ ${ }^{4}$ said57.rifai@gmail.com, ${ }^{5}$ rhali.benamar@gmail.com
}

Received 31 December 2020; received in revised form 24 March 2021; accepted 18 April 2021 DOI https://doi.org/10.21595/jve.2021.21857

Check for updates

Copyright $(2021$ Omar Outassafte, et al. This is an open access article distributed under the Creative Commons Attribution License, which permits unrestricted use, distribution, and reproduction in any medium, provided the original work is properly cited.

\begin{abstract}
The purpose of this present work is to investigate the geometrical non-linearity in free and forced vibration of a shallow arch elastically restrained at the ends. The non-linear governing equilibrium equation of the shallow arch is obtained after the Euler Bernoulli theory and the Von Karman geometrical non-linearity assumptions. After applying the ends conditions, the eigenvalues problem of the generalized trancendant equation have been determined iteratively using the Newton-Raphson algorithm. The kinetic and total strain energy have been discretized into a series of a finite spatial functions which are a combination of linear modes and basic function contribution coefficients. Using Hamilton's principle energy and spectral analysis, the problem is reduced into a set of non-linear algebraic equations that solved numerically using an approximate explicit method developed previously the so-called second formulation. Considering a multimode approach, the effect of initial rise and concentrated force on non-linear behaviour of system has been illustrated in the backbone curves giving the non-linear amplitude-frequency dependence. The corresponding non-linear deflections and curvatures have been plotted for various vibration amplitudes.
\end{abstract}

Keywords: free and forced vibration, shallow arch, Newton-Raphson, Hamilton's principle, second formulation, initial rise, backbone curves.

\section{Introduction}

Investigating the geometrical non-linearity is one of the major consideration on the design process of the arches. The study of the geometrical non-linearity of a beams, plates ,shells and circular arches were investigated by the authors of [1-8]. The authors classified the arches following their shallowness ratio into two classes; shallow arches and deep or no-shallow arches. The present study will be mainly focused on the non-linear free and forced vibration of a sinusoidal shallow arch.

Many researchers have studied the static and dynamic of shallow arch, experimentally and theoretically. Raymond H. Plaut et al. [9] determined the form of the arch which maximizes the fundamental vibration frequency.

Nayfeh et al. [10] obtained an exact solution of the eigenvalue problems governing linear undumped free vibrations of buckled beam, W. Lacarbona and G. Rega [11] obtained the general conditions for orthogonality of the non-linear modes in case of two-to-one, two-to-three, and one-to-one resonance. Using the multiple scales method, Yi Z., Stanciulescu [12] constructed the NNMs by considering the quadratic and cubic nonlinearities for a shallow arch with one end elastically restrained in vertical and rotational directions in the case of two-to-one internal resonances.

George C. Tsiatas and Nick G. Babouskos [13] investigated the linear and geometrically non-linear response of non-uniform shallow arches under a central concentrated force and they assume that the shallow arches have increased overall stiffness contrary to straight beams. The harmonic balance method and pseudo arc-length method was applied by Si-Qin Ye et al. [14] to 
study non-linear vibration of curved structure with non-linear boundary conditions. The author of [15] used a technique that combining harmonic balance coupled with continuation scheme to determine the solutions for an entire range of externally applied loading. The effects of a low frequency parametric excitation for a double hinged shallow arch with lateral and sinusoidal chargement of arch have been investigated by F. Lakrad, W. Schiehlen [16]. E. Ozkaya et al were analyzed the effect of different elastic springs coefficients and locations on non-linear transverse vibration of curved beam using the multiple scale method [17]. All these authors confirmed that linear and non-linear frequencies and modes shapes are sensitive to the linear initial curvature.

In this present paper, the analyze of the geometrical non-linearity in free and forced vibration of a sinusoidal shallow arch elastically restrained at the ends, will be presented following the analogous of $[18,19]$. The effects of the parameters such as the initial curvature and concentrated force were examined. The theoretical model is based on the Euler-Bernoulli beam theory and the von Kármán geometrical non-linearity assumptions. Harmonic motion is assumed and the transverse displacement is expended into a series of finites spatial functions. Using Hamilton's principle energy, the problem is reduced into a set of non-linear algebraic equations solved numerically using an approximate method the so-called second formulation. Based on multimode approach, the non-linear behavior of system can be clearly observed in the backbone curves giving the non-linear amplitude-frequency dependence of the structure. The corresponding first and second non-linear deflections and curvatures of the arch are presented.

\section{Mathematical review}

\subsection{The governing equation}

In the preset study, an Euler-Bernoulli shallow arch with initial curvature $w_{0}(x)$, initial rise $q$ and elastically restrained at the ends by vertical and rotational springs at left and right with stiffness coefficients $K_{t_{1}}, K_{\theta_{1}}, K_{t_{2}}$ and $K_{\theta_{2}}$ respectively. The expressions of the kinetic energy $T$, the axial strain energy $V_{a}$ and the bending strain energy $V_{b}$ of the system represented in Fig. 1 may be written as:

$$
\begin{aligned}
& T=\frac{1}{2} \rho A \int_{0}^{L}\left(\frac{\partial w(x, t)}{\partial t}\right)^{2} d x \\
& V_{a}=\frac{1}{2} E A\left[\int_{0}^{L}\left(\frac{d w_{0}(x)}{d x} \frac{\partial w(x, t)}{\partial x}+\frac{1}{2}\left(\frac{\partial w(x, t)}{\partial x}\right)^{2}\right) d x\right]^{2}, \\
& V_{b}=\frac{1}{2} E I \int_{0}^{L}\left(\frac{\partial^{2} w(x, t)}{\partial x^{2}}\right)^{2} d x .
\end{aligned}
$$

The total strain energy of a shallow $\operatorname{arch} V$ can be expressed as the sum of the axial strain energy $V_{a}$ and the bending strain energy $V_{b}$ as:

$V=V_{a}+V_{b}$

where $L$ is the projected length of the beam, A cross-section, $\rho$ is the density per unit length, $I$ the moment of inertia, $E$ the modulus of elasticity, $w_{0}(x)$ is the initial configuration function which is assumed to be:

$w_{0}(x)=\frac{q}{2}\left(1-\cos \left(\frac{2 \pi x}{L}\right)\right)$. 


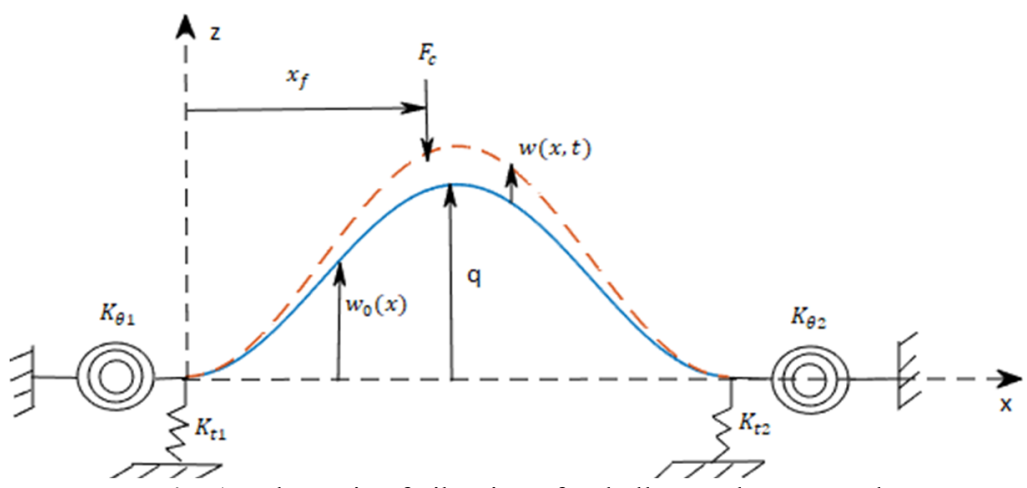

Fig. 1. Schematic of vibration of a shallow arch supported by translational and rotational springs at both ends

The kinetic energy Eq. (1) and total strain energy Eq. (4) due to the bending and axial strain are used in Hamilton's principle as defined in Eq. (5) to obtain the equilibrium equation of motion of a shallow arch, the longitudinal displacement is eliminated from the equations:

$\partial \int_{t_{1}}^{t_{2}}(V-T) d t=0$

The corresponding equation of motion can be written as follows:

$$
\begin{aligned}
\rho A & \frac{\partial^{2} w(x, t)}{\partial t^{2}}+E I \frac{\partial^{4} w(x, t)}{\partial x^{2}} \\
& =\frac{E A}{2 L}\left(\frac{\partial^{2} w(x, t)}{\partial x^{2}}+\frac{d^{2} w_{0}(x)}{d x^{2}}\right) \int_{0}^{L}\left(\left(\frac{\partial w(x, t)}{\partial x}\right)^{2}+2 \frac{d w_{0}(x)}{d x} \frac{\partial w(x, t)}{\partial x}\right) d x .
\end{aligned}
$$

For convenience, the following non-dimensional variables are used:

$$
x^{*}=\frac{x}{L}, \quad w^{*}=\frac{w}{r}, \quad w_{0}^{*}=\frac{w_{0}}{r}, \quad t=t^{*} \sqrt{\frac{E I}{\rho A L^{4}}}, \quad q^{*}=\frac{q}{r},
$$

where $r=\sqrt{I / A}$ is the radius of gyration of the cross section, $x^{*}$ is the dimensionless length, $w^{*}(x)$ is the dimensionless deflection from the initial configuration, $w_{0}{ }^{*}(x)$ the dimensionless initial curvature of the arch., $q^{*}$ is the dimensionless initial rise of the arch. The dimensionless equation of motion can be written as follows:

$$
\begin{aligned}
& \frac{\partial^{2} w(x, t)}{\partial t^{2}}+\frac{\partial^{4} w(x, t)}{\partial x^{2}} \\
& \quad=\left(\frac{\partial^{2} w(x, t)}{\partial x^{2}}+\frac{d^{2} w_{0}(x)}{d x^{2}}\right) \int_{0}^{1}\left(\left(\frac{\partial w(x, t)}{\partial x}\right)^{2}+2 \frac{d w_{0}(x)}{d x} \frac{\partial w(x, t)}{\partial x}\right) d x
\end{aligned}
$$

The stars of the dimensionless parameter of equations are vanished to simplify their rewriting. The boundary conditions of system at left rand at right and are respectively.

The left end condition: 
$\left.\frac{d^{3} w^{*}\left(x^{*}\right)}{d x^{* 3}}\right|_{x^{*}=0}=-\left.K_{t_{1}} w^{*}\left(x^{*}\right)\right|_{x^{*}=0}=0,\left.\quad \frac{d^{2} w^{*}\left(x^{*}\right)}{d x^{* 2}}\right|_{x^{*}=0}=\left.K_{\theta_{1}} \frac{d w^{*}\left(x^{*}\right)}{d x^{*}}\right|_{x^{*}=0}=0$.

The right end condition:

$\left.\frac{d^{3} w^{*}\left(x^{*}\right)}{d x^{* 3}}\right|_{x^{*}=1}=\left.K_{t_{2}} w^{*}\left(x^{*}\right)\right|_{x^{*}=1}=0,\left.\quad \frac{d^{2} w^{*}\left(x^{*}\right)}{d x^{* 2}}\right|_{x^{*}=1}=-\left.K_{\theta_{2}} \frac{d w^{*}\left(x^{*}\right)}{d x^{*}}\right|_{x^{*}=1}=0$.

\subsection{Linear problem}

The linear mode shapes and frequencies satisfy the following differential system:

$\frac{\partial^{2} w(x, t)}{\partial t^{2}}+\frac{\partial^{4} w(x, t)}{\partial x^{2}}=\frac{d^{2} w_{0}(x)}{d x^{2}} \int_{0}^{L}\left(2 \frac{d w_{0}(x)}{d x} \frac{\partial w(x, t)}{\partial x}\right) d x$

The motion is assumed to be harmonic $w(x, t)=w(x) e^{i \omega t}$ where $i=(-1)^{\frac{1}{2}}, \omega$ is the linear frequency and $w(x)$ is the mode shape function. The linear differential equation of motion becomes such bellow:

$\frac{d^{4} w(x)}{d x^{4}}-\omega^{2} w(x)=\frac{d^{2} w_{0}(x)}{d x^{2}} \int_{0}^{1} \frac{d w(x)}{d x} \frac{d w_{0}(x)}{d x} d x$.

The solution of the differential Eq. (13), may be written as a sum of homogenous a particular solution as [10]:

$w(x)=c_{1} \cosh \left(\omega^{\frac{1}{2}} x\right)+c_{2} \sinh \left(\omega^{\frac{1}{2}} x\right)+c_{3} \cos \left(\omega^{\frac{1}{2}} x\right)+c_{4} \sin \left(\omega^{\frac{1}{2}} x\right)+c_{5} \cos (2 \pi x)$.

The eigenvalues system $5 \times 5$ has been determined iteratively using Newton-Raphson algorithm.

The first five frequencies dependent to the initial rise of the arch have been plotted in Fig. 2 by assuming the vertical and rotational stiffness $K_{t_{1}}=K_{\theta_{1}}=K_{t_{2}}=K_{\theta_{2}}=\infty$ and considering the initial rise of the $q$ varies in interval $[0,20]$.

In Fig. 3 and 4 the first eight symmetrical and anti-symmetrical modes shapes are plotted respectively for a value of initial rise $q=4$.

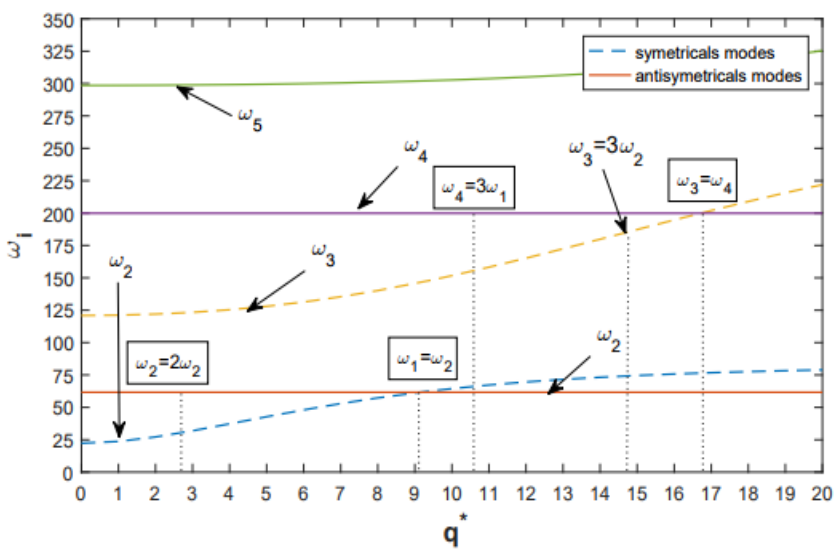

Fig. 2. Variation of the natural frequencies of the five modes of clamped-clamped shallow arch with nondimensional rise, dash line (- -) for symmetrical modes, continuous line is for antisymmetrical modes 
The Fig. 2 shows the variation of the frequencies with the initial dimensionless rise of the arch. We noticed that only the symmetrical mode influenced by the initial rise dimensionless of the arch. The crossing phenomena between two approaching (first order and second order modes) exists at $q=9.168$ and (third-order and fourth-order modes) at $q=16.713$. The results are compared with those given by W. Lacarbonara and G. Rega in [11] and have a very good agreement as presented in Table 1. The first four linear symmetrical and antisymmetric mode shapes of the shallow arch are plotted respectively in Fig. 3 and 4 for an initial value $q=4$.

Table 1. The ratios frequencies of clamped-clamped shallow arch

\begin{tabular}{|c|c|c|c|}
\hline Ratios & Frequencies & Present $q$ & Ref. [3] $q$ \\
\hline $1: 1$ & $\omega_{1}=\omega_{2}$ & 9.168 & 9.15 \\
\hline $1: 1$ & $\omega_{3}=\omega_{4}$ & 16.713 & 16.72 \\
\hline $2: 1$ & $\omega_{2}=2 \omega_{1}$ & 2.788 & 2.79 \\
\hline $2: 1$ & $\omega_{3}=2 \omega_{2}$ & 3.01 & 3.01 \\
\hline $3: 1$ & $\omega_{3}=3 \omega_{1}$ & 4.975 & 4.98 \\
\hline $3: 1$ & $\omega_{4}=3 \omega_{1}$ & 10.782 & 10.78 \\
\hline $3: 1$ & $\omega_{3}=3 \omega_{2}$ & 14.712 & 14.70 \\
\hline
\end{tabular}

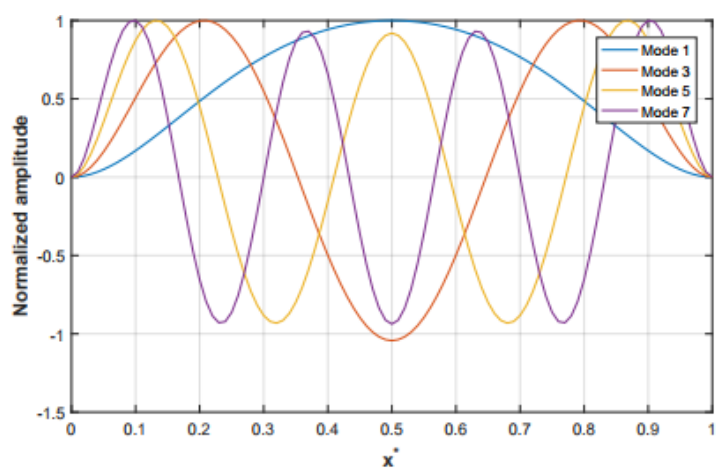

Fig. 3. Symmetrical linear displacement $w_{i}$ for $i=1,3,5$ and 7 for an initial rise $q=4$

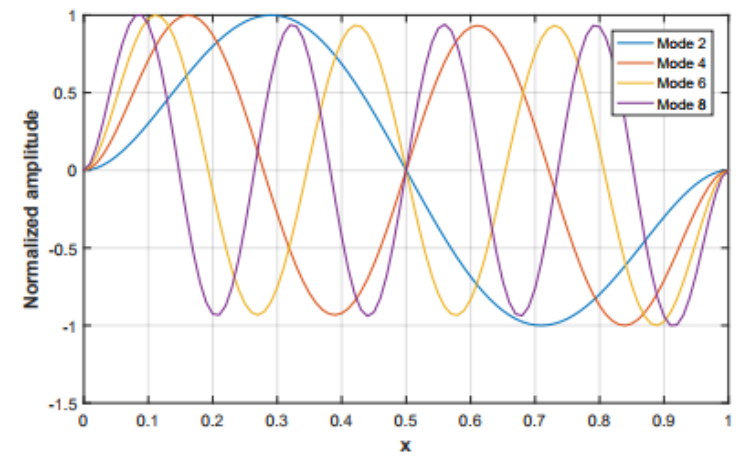

Fig. 4. Anti- symmetrical linear displacement $w_{i}$ for $i=2,4,6$ and 8 for an initial rise $q=4$

\subsection{Formulation of the non-linear problem}

To develop the non-linear theory, the transverse displacement function is expanded as a series of $N$ basic spatial functions:

$w(x, t)=q_{i}(t) w_{i}(x)=a_{i} w_{i} \sin (\omega t)$.

Using a generalized parameterization and the usual summation convention defined in $[4,18]$, 
the kinetic energy, and the total strain energy due to axial and bending strain energy of the shallow arch can be expressed as:

$T=\frac{1}{2} \omega^{2} a_{i} a_{j} m_{i j} \cos ^{2}(\omega t)$,

$V_{a}=\frac{1}{2} a_{i} a_{j} k_{i j} \sin ^{2}(\omega t)$,

$V_{b}=\frac{1}{2} a_{i} a_{j} a_{k} C_{i j k} \sin ^{3}(\omega t)+\frac{1}{2} a_{i} a_{j} a_{k} a_{l} b_{i j k l} \sin ^{4}(\omega t)$.

where $m_{i j}, k_{i j}, C_{i j k}$ and $b_{i j k l}$ are respectively, the mass tensor, linear, cubic nonlinear and quadratic nonlinear rigidity of the shallow arch in which their expressions are defined as:

$$
\begin{aligned}
& m_{i j}=\rho A \int_{0}^{L} w_{i}(x) w_{j}(x) d x \\
& k_{i j}=E I \int_{0}^{L} \frac{\partial^{2} w_{i}}{\partial x^{2}} \frac{\partial^{2} w_{j}}{\partial x^{2}} d x+\frac{E A}{L} \int_{0}^{L} \frac{\partial w_{i}}{\partial x} \frac{d w_{0}}{d x} d x \int_{0}^{L} \frac{\partial w_{j}}{\partial x} \frac{d w_{0}}{d x} d x \\
& C_{i j k}=\frac{E A}{L} \int_{0}^{L} \frac{\partial w_{i}}{\partial x} \frac{\partial w_{j}}{\partial x} d x \int_{0}^{L} \frac{\partial w_{k}}{\partial x} \frac{\partial w_{0}}{\partial x} d x \\
& b_{i j k l}=\frac{E A}{4 L} \int_{0}^{L} \frac{\partial w_{i}}{\partial x} \frac{\partial w_{j}}{\partial x} d x \int_{0}^{L} \frac{\partial w_{k}}{\partial x} \frac{\partial w_{l}}{\partial x} d x .
\end{aligned}
$$

The coefficients $a_{i}$ are unknowns as well as the frequency $\omega_{i}$. The dynamic behavior of the beam is examined under a centered point force $F_{c}$ applied at point $x_{f}$ and a distributed harmonic force $F_{d}$ defined by:

$F_{d}(x, t)=f^{d} \sin (\omega t)$.

$\delta$ is the Dirac function, and $F_{i}^{c}(t)$ and $F_{i}^{d}(t)$ are the corresponding concentrated force and generalized forces that expressed as:

$$
\begin{aligned}
& F_{i}^{c}(t)=F^{c} \sin (\omega t) w_{i}\left(x_{0}\right) d x=f_{i}^{c} \sin (\omega t), \\
& F_{i}^{d}(t)=F^{d} \sin (\omega t) \int_{0}^{1} w_{i}(x) d x=f_{i}^{d} \sin (\omega t) .
\end{aligned}
$$

Using Hamilton's principle defined in Eq. (5) and the matrix notation by taking into account the forcing term, the process leads to non-linear algebraic equations for the unknown coefficients $a_{i}$ that can be written in a matrix form as follows:

$$
\left([K]-\omega^{2}[M]\right)\{A\}+\frac{3}{2}[B(A)]\{A\}=\{F\},
$$

where $\{A\}$ is a column vector of the basic functions contribution coefficients $a_{i}$. To evaluate the non-dimensional parameter, ones put:

$$
w_{i}(x)=h w_{i}^{*}\left(\frac{x}{L}\right)=h w_{i}^{*}\left(x^{*}\right), \quad \frac{m_{i j}}{m_{i j}^{*}}=\rho A h^{2} L, \quad \frac{k_{i j}}{k_{i j}^{*}}=\frac{C_{i j k}}{C_{i j k}^{*}}=\frac{b_{i j k l}}{b_{i j l}^{*}}=\frac{E I h^{2}}{L^{3}} .
$$


And the dimensionless generalized forces $f_{i}^{c *}$ and $f_{i}^{d *}$ are given by:

$f_{i}^{* c}=F^{c} \frac{R^{3}}{E \operatorname{Ih}^{2}} w_{i}\left(x_{f}^{*}\right), \quad f_{i}^{* d}=F^{d} \frac{L^{4}}{E I h} \int_{0}^{1} w_{i}^{*}\left(x^{*}\right) d x^{*}$.

Which leads to:

$\omega=\frac{E I}{\rho A L^{4}}$

where $m_{i j}^{*}, k_{i j}^{*}, C_{i j k}^{*}$ and $b_{i j k l}^{*}$ are the non-dimensional generalized parameters given by:

$m_{i j}^{*}=\int_{0}^{1} w_{i}^{*}(x) w_{j}^{*}(x) d x^{*}$

$k_{i j}{ }^{*}={ }^{*} \int_{0}^{1} \frac{\partial w_{i}{ }^{*}}{\partial x^{*}} \frac{d w_{0}{ }^{*}}{d x^{*}} d x^{*} \int_{0}^{1} \frac{\partial w_{j}^{*}}{\partial x^{*}} \frac{d w_{0}^{*}}{d x^{*}} d x^{*}+\int_{0}^{1} \frac{\partial^{2} w_{i}^{*}}{\partial x^{* 2}} \frac{\partial^{2} w_{j}^{*}}{\partial x^{* 2}} d x$,

$C_{i j k}^{*}=\int_{0}^{1} \frac{\partial w_{i}^{*}}{\partial x^{*}} \frac{\partial w_{j}^{*}}{\partial x^{*}} d x^{*} \int_{0}^{1} \frac{\partial w_{k}^{*}}{\partial x^{*}} \frac{\partial w_{0}^{*}}{\partial x^{*}} d x^{*}$,

$b_{i j k l}=\alpha \int_{0}^{1} \frac{\partial w_{i}^{*}}{\partial x^{*}} \frac{\partial w_{j}^{*}}{\partial x^{*}} d x^{*} \int_{0}^{1} \frac{\partial w_{k}^{*}}{\partial x^{*}} \frac{\partial w_{l}^{*}}{\partial x^{*}} d x^{*}$.

For a uniform rectangular cross-section, $\alpha=3$, since in this case, $\left(\frac{h^{2} A}{I}=12\right)$. The functions $\left\{w_{i}^{*}, i=1, \ldots, n\right\}$ have been normalized in order to obtain the mass matrix equals the identity matrix. The corresponding integrals, defined in Eqs. (29)-(32), have been calculated numerically, using the Simpson's rule. Eq. (25) can be rewritten in non-dimensional form as:

$\left(\left[K^{*}\right]-\omega^{2}\left[M^{*}\right]\right)\{A\}+\frac{3}{2}\left[B^{*}(A)\right]\{A\}=\left\{F^{*}\right\}$.

Using the tensorial notation, ones put:

$a_{i} k_{i r}^{*}+\frac{3}{2} a_{i} a_{j} a_{k} b_{i j k r}^{*}-a_{i} \omega^{* 2} m_{i r}^{*}=f_{r}^{c *}, \quad r=1, \ldots, n$.

Eq. (33) represents a set of nonlinear algebraic equations in which $\left\{F^{*}\right\}$ is a column vector of generalized forces. This equation appears as a generalization to the non-linear case of the classical linear forced response equation, very well known in linear modal analysis theory, to which the term $\frac{3}{2}\left[B^{*}(\{A\})\right]\{A\}$ corresponding to the non-linear geometrical rigidity is added.

The parameters $m_{i j}^{*}, k_{i j}^{*}$ and $b_{i j k l}^{*}$ have been solved numerically using Simpson's method in range $[0,1]$ and the non-linear algebraic Eq. (34) has been solved using the formulation developed in [10] that called the second formulation, it is an approximation which consists to separate the nonlinear term of Eq. (34) into a terms proportional to $a_{1}^{3}$ and terms proportional to $a_{1}^{2} \varepsilon_{i}$ and neglecting terms proportional to $a_{1} \varepsilon_{i} \varepsilon_{j}$ and terms proportional to $\varepsilon_{i} \varepsilon_{j} \varepsilon_{k}$ one can write:

$a_{i} a_{j} a_{k} b_{i j k r}=a_{1}^{3} b_{111 r}+a_{1}^{2} \varepsilon_{i} b_{11 i r}, \quad r=1, \ldots, n$. 
After substituting and rearranging, Eq. (35) can be written in matrix form as:

$$
\left(\left[K_{r}^{*}\right]_{R}-\omega^{2}\left[M_{r}^{*}\right]_{R}\right)\left\{A_{r}\right\}_{R}+\frac{3}{2}\left[\alpha^{*}{ }_{r}\right]_{R}\left\{A_{r}\right\}_{R}=\left\{F_{r}^{*}-\frac{3}{2} a_{r}^{3} b_{111 r}^{*}\right\} .
$$

In which $\left[K_{r}{ }^{*}\right]_{R}=\left[k_{i j}{ }^{*}\right]_{R}$ and $\left[M_{r}{ }^{*}\right]_{R}=\left[m_{i j}{ }^{*}\right]_{R}$ are reduced rigidity and mass matrices associated with the first nonlinear mode, obtained by varying $i$ and $j,\left[\alpha_{r}{ }^{*}\right]_{R}$ is a square matrix, depending on $a_{1}$, whose general term $\alpha_{i j}{ }^{*}$ is equal to $a_{1}{ }^{2} b_{11 i r}$, and $a_{1}^{3} b_{111 r}$ is a column vector representing the right side of the linear system Eq. (32) in which the reduced unknown vector is $\left\{A_{r}\right\}_{R}=\left\{\varepsilon_{2}, \varepsilon_{3}, \ldots, \varepsilon_{r}\right\}^{T}$.

The modal contributions $\varepsilon_{i}$ can be obtained easily by solving the non-linear system Eq. (35) of $n$ equations and $n$ unknowns. Higher nonlinear mode shapes may be obtained in a similar manner, using appropriate reduced matrix in each case.

Where the frequency ratio $\omega^{* 2}$ is given from Eq. (34):

$\left(\frac{\omega_{n l}^{*}}{\omega_{l}^{*}}\right)^{2}=\frac{3}{2} \frac{b_{i i i i}^{*}}{k_{i i}^{*}} a_{i}^{2}-\frac{f_{i}^{* c}}{a_{i} k_{i i}^{*}}+1, \quad i=1, \ldots, n$,

where $\omega_{l}^{*}$ is the linear natural frequency parameter that can be written as:

$\left(\omega_{l}^{*}\right)^{2}=\frac{k_{i i}^{*}}{m_{i i}^{*}}, \quad i=1, \ldots, n$.

If free vibration case is considered the dependence of the non-linear frequency ratio $\frac{\omega_{n l}^{*}}{\omega_{l}^{*}}$ on the dimensionaless vibration amplitude $w_{\text {max }}^{*}$ is obtained from Eq. (37) by putting $f_{i}^{* c}=0$. The normalized first nonlinear mode shape for a given assigned value $a_{1}$ is then obtained as a series involving the arch modal parameters depending on the first six symmetric modes given by [18]:

$W_{n l 1}^{*}\left(x, a_{1}\right)=a_{1} w_{1}^{*}(x)+\varepsilon_{3} w_{3}^{*}(x)+\varepsilon_{5} w_{5}^{*}(x)+\varepsilon_{7} w_{7}^{*}(x)+\varepsilon_{9} w_{9}^{*}(x)+\varepsilon_{11} w_{11}^{*}(x)$.

In which the predominant term, proportional to the first linear mode shape, is $a_{1} w_{1}^{*}(x)$ and other terms, proportional to the higher modes $w_{3}^{*}(x), \ldots, w_{11}^{*}(x)$ are the corrections due to the nonlinearity. For the second amplitude-dependent non-linear mode shape $w_{n l 2}^{*}\left(x, a_{2}\right)$ for a given assigned value $a_{2}$ of the second function contribution, the predominant term is $a_{2} w_{2}^{*}(x)$ and other terms, proportional to the higher modes $w_{4}^{*}(x), \ldots, w_{12}^{*}(x)$ are the corrections due to the nonlinearity.

\section{Results and discussion}

In this section, numerical results obtained for various values of the initial rise and levels of the excitation force will be presented via solution of the set of non-linear algebraic equations Eq. (36), based on the second formulation for the value of the stiffness of the translational and rotational springs at both ends $K_{t_{1}}=K_{t_{2}}=K_{\theta_{1}}=K_{\theta_{2}}=\infty$.

The basic functions contributions $\varepsilon_{i}$ for $i$ th mode shapes, the corresponding maximum nondimensional vibration amplitudes, and the curvatures at $x=0$ are given in Table 2 and 3 for each given value of $a_{i}$. It can be seen in this tables, that the contribution $a_{i}$ remains predominant for the whole range of vibration amplitudes considered, compared to the contributions $\varepsilon_{i}$ of the other functions, it can be noticed also that the contribution coefficients $\varepsilon_{i}$ increased according to the maximum non-dimensional amplitude $w_{\text {max }}^{*}$.

The numerical solutions obtained, have been substituted in Eq. (36) in case of $\left\{F_{r}^{*}\right\}=\{0\}$ and 
calculation has been made of the resulting vector $\{$ Residual\}.

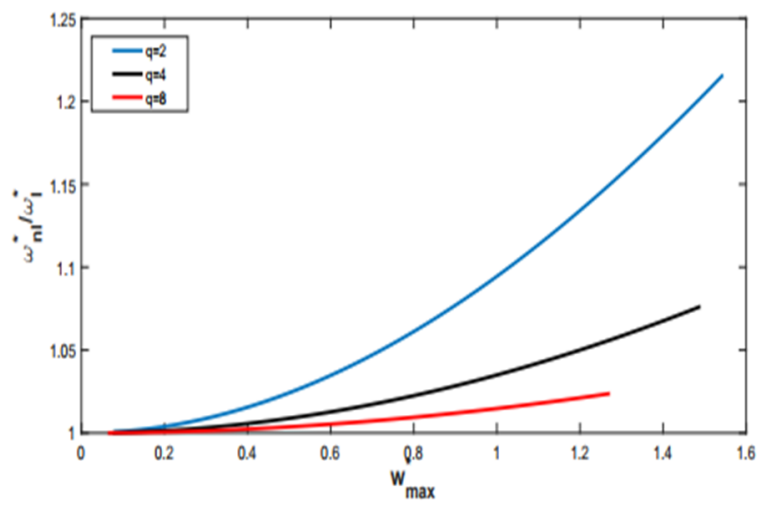

Fig. 5. Backbone curves of clamped-clamped shallow arch, in the vicinity of the first mode, for various values of initial rises $(q=2,4$ and 8$)$

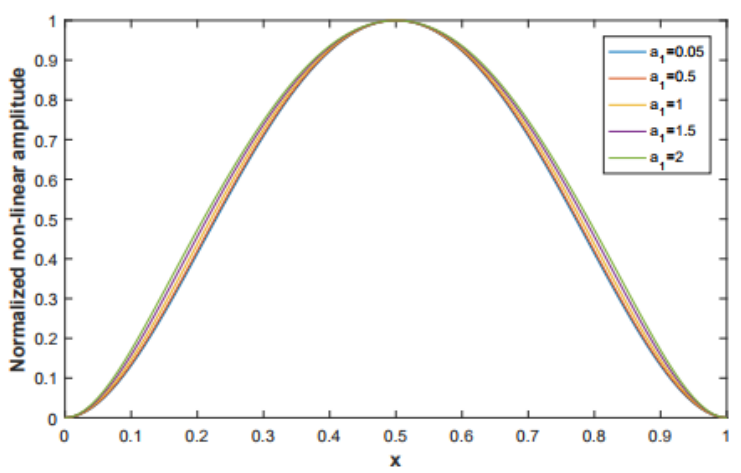

Fig. 6. The first normalized non-linear mode shape for $q=4$

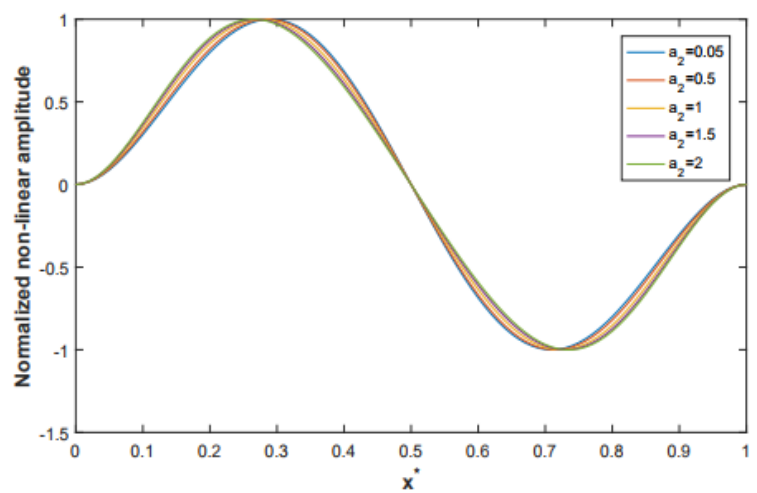

Fig. 7. The second normalized non-linear mode shape for $q=4$

In Fig. 5, the backbone curves giving the non-linear amplitude-frequency dependence, based on the multimode approach of a $\mathrm{C}-\mathrm{C}$ shallow arch are plotted in the neighborhood of the first nonlinear mode shape for various initial rise $q=2,4$ and 8 . It may be noticed that increasing the initial rise the nonlinearity decreases, these results are due to the fact that when the initial rise is increased, the flexural rigidity of the arch increases. We can also notice from the Fig. 2 that the linear frequencies of symmetrical modes increase by the increasing of the initial rise, consequently the frequency ratio decreases since the linear frequency presents the denominator of the frequency 
ratio. However, in the second vibration mode, the non-dimensional frequency ratios exhibit the same behavior as the straight beam. These results are compatible with the results of linear regime.

The normalized non-dimensional vibration amplitude corresponding to the first and second mode shape are presented respectively in Fig. 6 and 7.

In Fig. 8 and 9, the curvature curves of clamped-clamped shallow arch, associated to the first and second non-linear deflection are plotted for various levels of vibration amplitudes by taking into account an initial rise $q=4$.

Table 2. Frequency ratios of free vibration in the first non-linear mode, curvatures and modal participation of a C-C shallow arch at various amplitudes obtained by the second formulation

\begin{tabular}{|c|c|c|c|c|c|c|c|c|c|}
\hline$\frac{\omega_{n l}^{*}}{\omega_{l}^{*}}$ & $w_{\max }^{*}$ & $\frac{d^{2} w}{d x^{2}}(0)$ & $a_{1}$ & $\varepsilon_{3}$ & $\varepsilon_{5}$ & $\varepsilon_{7}$ & $\varepsilon_{9}$ & $\varepsilon_{11}$ & Residual \\
\hline 1.000198 & 0.074687 & 2.608599 & 0.05 & $1,11 \mathrm{E}-06$ & $5,22 \mathrm{E}-07$ & $1,17 \mathrm{E}-07$ & $3,60 \mathrm{E}-08$ & $1,72 \mathrm{E}-08$ & $8.43 \mathrm{E}-35$ \\
\hline 1.019601 & 0.745852 & 26.66276 & 0.5 & 0,00103 & 0,000506 & 0,000117 & $3,63 \mathrm{E}-05$ & $1,73 \mathrm{E}-05$ & $3.79 \mathrm{E}-29$ \\
\hline 1.076265 & 1.48718 & 56.22632 & 1 & 0,006837 & 0,003686 & 0,00092 & 0,000295 & 0,000139 & $7.47 \mathrm{E}-27$ \\
\hline 1.164595 & 2.223823 & 89.63892 & 1.5 & 0,018144 & 0,010791 & 0,002977 & 0,001 & 0,000467 & $1.49 \mathrm{E}-25$ \\
\hline
\end{tabular}

Table 3. Frequency ratios of free vibration in the second non-linear mode, curvatures and modal participation of a C-C shallow arch at various amplitudes obtained by the second formulation

\begin{tabular}{|c|c|c|c|c|c|c|c|c|c|}
\hline$\frac{\omega_{n l}^{*}}{\omega_{l}^{*}}$ & $w_{\max }^{*}$ & $\frac{d^{2} w}{d x^{2}}(0)$ & $a_{2}$ & $\varepsilon_{2}$ & $\varepsilon_{4}$ & $\varepsilon_{6}$ & $\varepsilon_{8}$ & $\varepsilon_{12}$ & Residual \\
\hline 1.002089 & 0.0754 & 6.176212 & 0.05 & $2.24 \mathrm{E}-05$ & $4.59 \mathrm{E}-06$ & $1.23 \mathrm{E}-06$ & $5.46 \mathrm{E}-07$ & $-3.65 \mathrm{E}-08$ & $4.62 \mathrm{E}-32$ \\
\hline 1.190862 & 0.7470 & 68.37282 & 0.5 & 0.016765 & 0.004105 & 0.00122 & 0.000563 & $-4.26 \mathrm{E}-05$ & $4.85 \mathrm{E}-26$ \\
\hline 1.634812 & 1.4742 & 154.2536 & 1 & 0.077353 & 0.02398 & 0.008552 & 0.004223 & -0.00041 & $2.10 \mathrm{E}-24$ \\
\hline 2.182516 & 2.1932 & 247.0735 & 1.5 & 0.155322 & 0.055208 & 0.022587 & 0.011836 & -0.0015 & $4.24 \mathrm{E}-23$ \\
\hline
\end{tabular}

Table 4. Comparison of the first non-linear frequency ratio $\frac{\omega_{n l}^{*}}{\omega_{l}^{*}}$ and maximal non-linear amplitude with those obtained in reference [4]

\begin{tabular}{|c|c|c|c|c|c|c|c|c|}
\hline & \multicolumn{2}{|c|}{ Ref. [4]. } & \multicolumn{2}{c|}{ Present with $q=2$} & \multicolumn{2}{|c|}{ Present with $q=4$} & \multicolumn{2}{|c|}{ Present with $q=8$} \\
\hline$a_{1}$ & $\frac{\omega_{n l}^{*}}{\omega_{l}^{*}}$ & $W_{\max }^{*}$ & $\frac{\omega_{n l}^{*}}{\omega_{l}^{*}}$ & $W_{\max }^{*}$ & $\frac{\omega_{n l}^{*}}{\omega_{l}^{*}}$ & $W_{\max }^{*}$ & $\frac{\omega_{n l}^{*}}{\omega_{l}^{*}}$ & $W_{\max }^{*}$ \\
\hline 0.05 & 1.001 & 0.079 & 1.00059 & 0.07824 & 1.0001 & 0.0746 & 1.00006 & 0.0636 \\
\hline 0.5 & 1.106 & 0.789 & 1.0581 & 0.7792 & 1.0196 & 0.7462 & 1.0059 & 0.6369 \\
\hline 1 & 1.361 & 1.554 & 1.2159 & 1.5432 & 1.0762 & 1.489 & 1.0237 & 1.2760 \\
\hline 1.5 & 1.684 & 2.297 & 1.441 & 2.2905 & 1.1645 & 2.228 & 1.0527 & 1.9175 \\
\hline
\end{tabular}

Table 5. Comparison of the second non-linear frequency ratio $\frac{\omega_{n l}^{*}}{\omega_{l}^{*}}$ and maximal non-linear amplitude with those obtained in reference [4]

\begin{tabular}{|c|c|c|c|c|c|c|c|c|}
\hline & \multicolumn{2}{|c|}{ Ref. [4]. } & \multicolumn{2}{c|}{ Present with $q=2$} & \multicolumn{2}{c|}{ Present with $q=4$} & \multicolumn{2}{c|}{ Present with $q=8$} \\
\hline$a_{2}$ & $\frac{\omega_{n l}^{*}}{\omega_{l}^{*}}$ & $W_{\max }^{*}$ & $\frac{\omega_{n l}^{*}}{\omega_{l}^{*}}$ & $W_{\max }^{*}$ & $\frac{\omega_{n l}^{*}}{\omega_{l}^{*}}$ & $W_{\max }^{*}$ & $\frac{\omega_{n l}^{*}}{\omega_{l}^{*}}$ & $W_{\max }^{*}$ \\
\hline 0.05 & 1.002 & 0.075 & 1.002 & 0.0754 & 1.002 & 0.0754 & 1.002 & 0.0754 \\
\hline 0.5 & 1.187 & 0.750 & 1.190 & 0.7470 & 1.190 & 0.7470 & 1.190 & 0.7493 \\
\hline 1 & 1.603 & 1.488 & 1.634 & 1.4741 & 1.634 & 1.4742 & 1.634 & 1.4809 \\
\hline 1.5 & 2.101 & 2.217 & 2.182 & 2.1946 & 2.182 & 2.1932 & 2.182 & 2.1992 \\
\hline
\end{tabular}




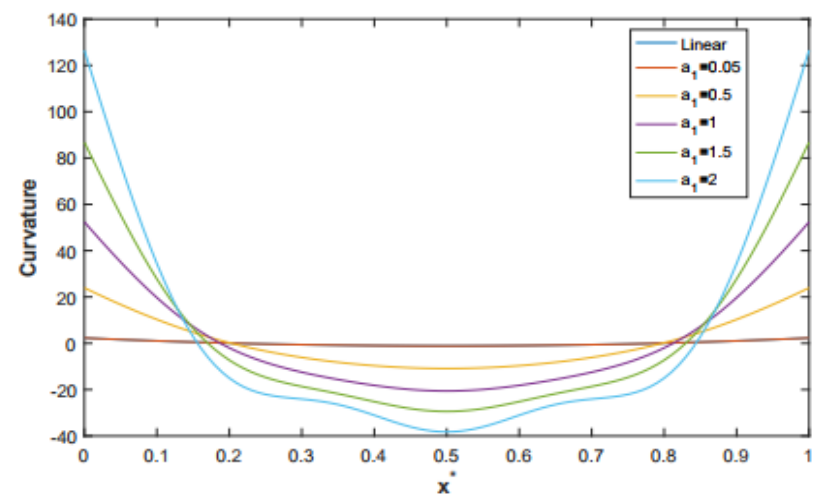

Fig. 8. The linear and non-linear curvature corresponding to the first mode shape for $q=4$

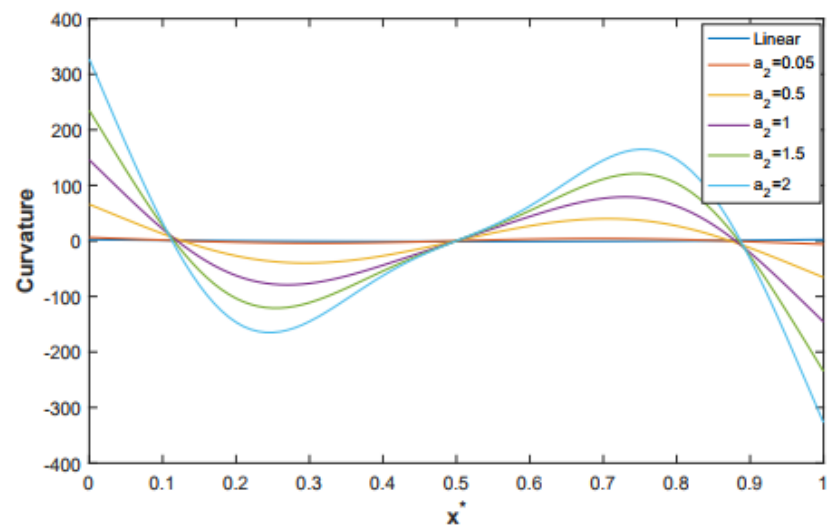

Fig. 9. The linear and non-linear curvatures corresponding to the second mode shape for $q=4$

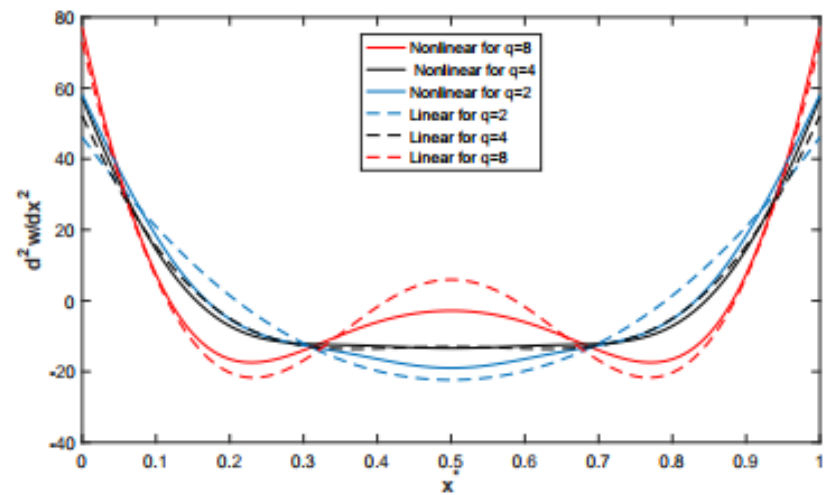

Fig. 10. Curvature distributions, associated to the first non-linear deflection, of a $\mathrm{C}-\mathrm{C}$ shallow arch, for various values of initial rise $(q=2,4$ and 8$)$ and a maximum non-dimensional amplitudes $\left(w_{\max }^{*}=1.5\right)$

The Table 4 and 5, present the values of the non-dimensional non-linear frequency ratios and the non-dimensional vibration amplitude of clamped-clamped shallow arch which are calculated using the second formulation for various values of the initial rise $q$. A comparison is made with a clamped-clamped beam straight beam [4].

The Table 5 shows that the frequency ratio remains constant along the variation of initial rise of the arch. In this case the same behavior of straight beam is may be observed. It was found, that for amplitude equal to 1 time the shallow arch thickness, the difference induced does not exceed 
$1.8 \%$ for the non-dimensional frequency ratio and $0.48 \%$ for the non-dimensional vibration in comparison with the results given by [4].

In Fig. 12 and 13, the non-linear frequency response functions of the shallow arch examined, are plotted in the neighborhood of the first non-linear mode shape, for various values of the concentrated force and initial rise $(q=2,4$ and 8$)$.

In Figs. 10, the curvature distributions are plotted, associated to the first linear and non-linear deflection, of a $\mathrm{C}-\mathrm{C}$ shallow arch in free vibration case, for various initial rise $(2,4,8)$ and a maximum non-dimensional amplitudes $\left(w_{\max }^{*}=1.5\right)$. The corresponding values are summarized in Table 6 , in which the percentage correction at the clamps and at the middles are given for various values of initial rise. An increase of curvature with the initial rise, especially at the clamped end of the shallow arch may be clearly observed.

Table 6 presents the calculation of the percentage correction between the curvatures via the nonlinear theory compared to the linear theory. it can be seen that the percentage correction at the middles of the arch reaches $17.74 \%$ for $q=2,2.91 \%$ for $q=4$, and $3.29 \%$ for $q=8$. In addition, it can be noticed that the maximum value of the linear and nonlinear curvature of the arch in case of $q=2,4$ and 8 is located near the clamped ends.

Table 6. Effect of rise on the percentage correction between the curvatures estimated via the linear and nonlinear theories for various values of initial rise. Maximum non-dimensional amplitudes $\left(w_{\max }^{*}=1.5\right)$

\begin{tabular}{|c|c|c|c|c|c|c|}
\hline \multirow{2}{*}{$q^{*}$} & \multicolumn{2}{|c|}{$\frac{d^{2} w^{*}}{d x^{* 2}}(0)$} & \multirow{2}{*}{$\begin{array}{c}\text { Percentage correction } \\
(\%)\end{array}$} & \multicolumn{2}{|c|}{$\frac{d^{2} w^{*}}{d x^{* 2}}\left(\frac{1}{2}\right)$} & \multirow{2}{*}{$\begin{array}{c}\text { Percentage correction } \\
(\%)\end{array}$} \\
\cline { 2 - 3 } & Linear & Nonlinear & & Linear & Nonlinear & \\
\hline 2 & 46.11 & 58.06 & 20.58 & -22.36 & -18.99 & 17.74 \\
\hline 4 & 52.15 & 57.30 & 8.98 & -13.00 & -13.39 & 2.91 \\
\hline 8 & 73.04 & 75.59 & 3.37 & 5.95 & -2.59 & 3.29 \\
\hline
\end{tabular}

Table. 7 presents the contributions of the six symmetric modes. The calculation of the generalized forces led to the conclusion that the first mode is predominant.

The hardening behavior type of geometrical non-linearity can be clearly observed in Fig. 11-13. The discontinuities shown in Fig. 11-15 are due to the fact of the existence of three amplitudes for one range of frequency, this phenomenon is characteristic on non-linear systems, and is called a jump phenomenon. Another property of non-linear system can be clearly observed; the frequency-response functions are not proportional to the level of the concentrated forces applied.

By assuming a multimode approach used previously in [18], Eq. (37) is plotted in the neighborhood of the first non-linear mode shape in case of various values of concentrated force $F^{*}$ in Fig. 12 and various values of the initial rise $q$ in Fig. 13. Figure 11 shows a comparison between the linear and nonlinear frequency response functions, where the shallow arch is excited by a concentrated force $F^{*}=100$.

Table 7. Percentage of generalized forces exciting the first six symmetric modes of a C-C shallow arch excited by uniformly distributed force over an interval $[0,1]$.

\begin{tabular}{|c|c|c|c|c|c|c|}
\hline Modes & 1 & 3 & 5 & 7 & 9 & 11 \\
\hline $\int_{0}^{1} w_{i}^{*}\left(x^{*}\right)$ & 0.6522 & 0.3572 & 0.2313 & 0.1697 & 0.1340 & 0.1095 \\
\hline$\frac{\int_{0}^{1} w_{i}^{*}\left(x^{*}\right)}{\sum_{i=1}^{12}\left|\int_{0}^{1} w_{i}^{*}\left(x^{*}\right)\right|}$ & 0.3943 & 0.2159 & 0.1398 & 0.1026 & 0.0810 & 0.0662 \\
\hline
\end{tabular}

In Fig. 12 and 13 the non-linear frequency response functions of the shallow arch are plotted in the neighborhood of the first non-linear mode shape for various levels of the concentrated force and initial rise $q$. The hardening type of geometrical non-linearity of the clamped-clamped shallow arch excited by a concentrated force is decreased in a remarkable way with increasing the initial 
rise. This is due to the fact that when the initial rise is increased, the flexural rigidity of the arch decreases.

The response curves have been illustrated for two cases: the first, the $\mathrm{C}-\mathrm{C}$ shallow arch was excited by a concentric harmonic excitation in Fig. 12 and 13, in the second, by a uniformly distributed force in Fig. 14 and 15. It may be observed from these figures, the hardening type of geometrical non-linearity also the jump phenomenon where for the same frequency range, several results are possible, as well as the non-proportional evolution of the frequency response to the intensity of excitation.

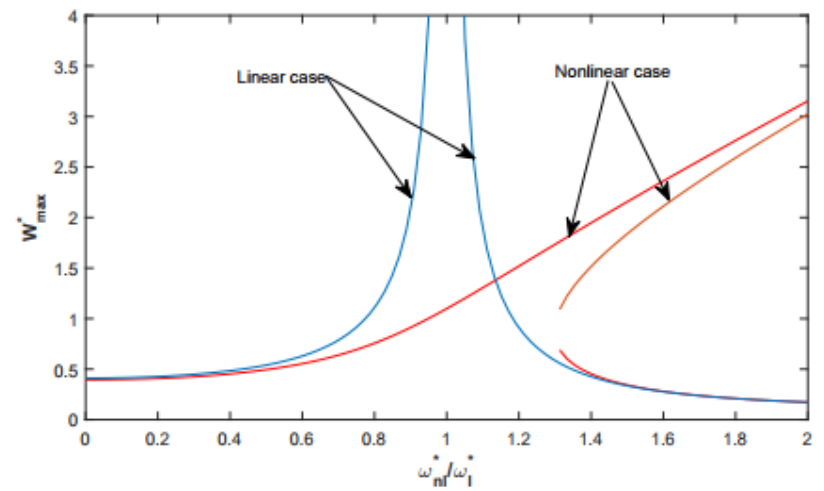

Fig. 11. Linear and nonlinear frequency response functions comparison for $F^{*}=100$ and $q=2$

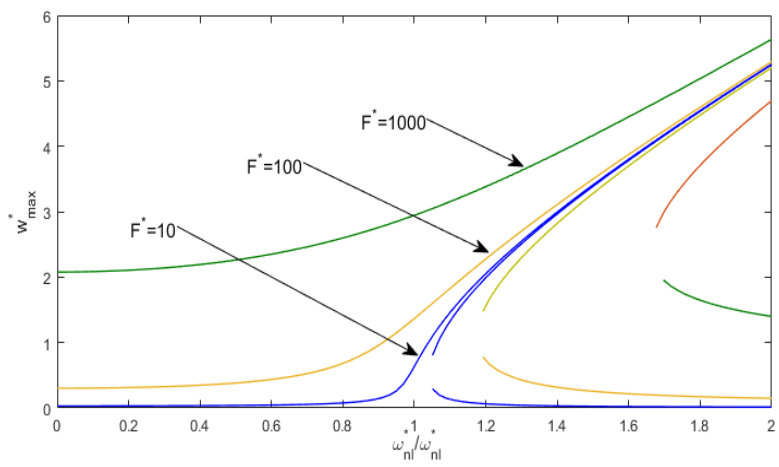

Fig. 12. Nonlinear frequency response functions, based on the multimode approach, of a $\mathrm{C}-\mathrm{C}$ shallow arch and various levels of the concentrated forces

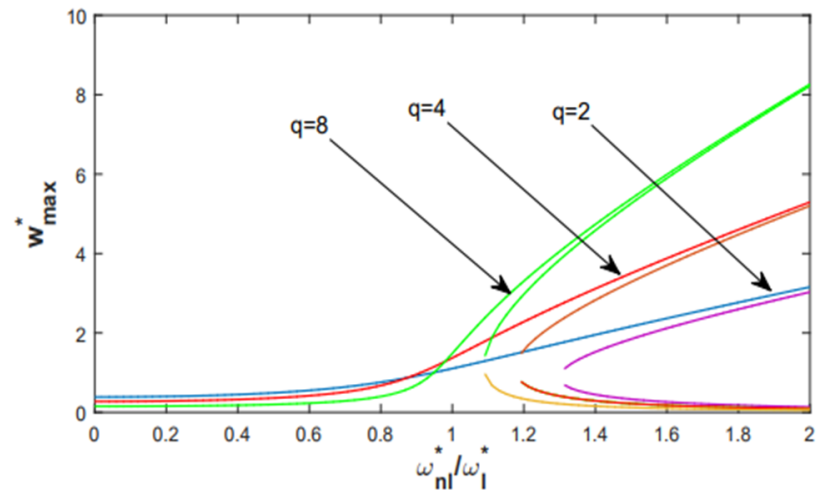

Fig. 13. Nonlinear frequency response functions, based on the multimode approach, of a C-C shallow arch for a concentrated forces $F^{*}=100$ and various levels of $q$ 


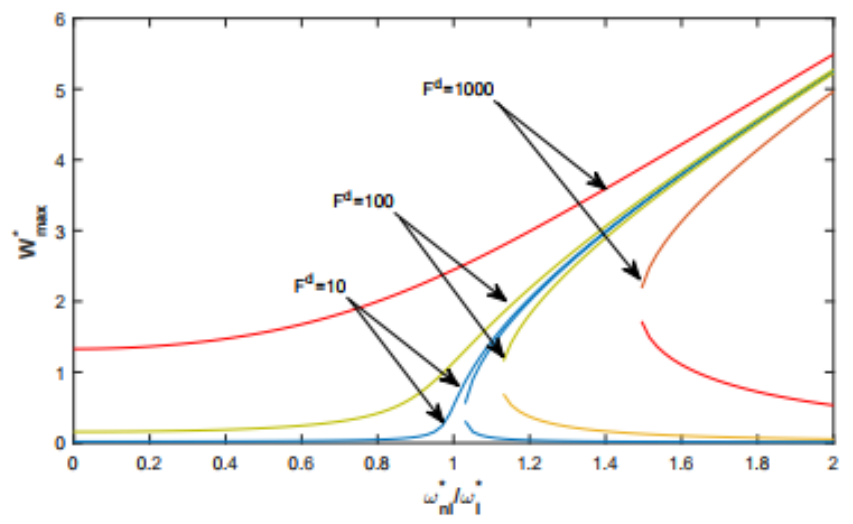

Fig. 14. Nonlinear frequency response functions. based on the multimode approach. of a C-C shallow arch and various levels of excitation: in case uniformly distributed harmonic force.

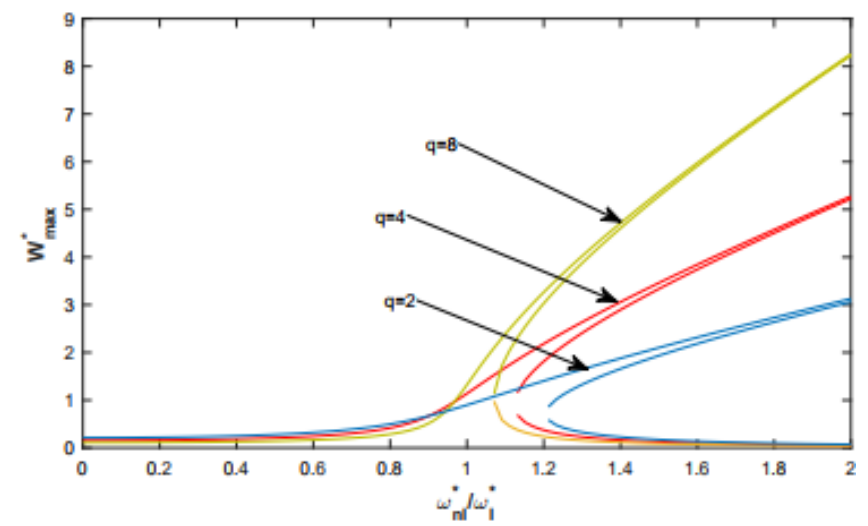

Fig. 15. Nonlinear frequency response functions. based on the multimode approach. of a C-C shallow arch for a harmonic force uniformly distributed $F^{d}=100$ and various levels of $q$.

\section{Conclusions}

A theoretical model to analyze the large vibration amplitude of shallow arch was presented to examine the geometrical non-linearity in transverse vibration of a shallow arch. The linear frequencies were obtained iteratively using the Newton-Raphson algorithm. The mathematical formulation of the problem is based on the Euler-Bernoulli theory and the Von Karman assumptions. The kinetic and total strain energy were derived and discretized into a series of a finites spatial functions by applying Hamilton's principle energy and spectral analysis. The problem was reduced into a set of non-linear algebraic equations solved numerically using an approximate method developed previously the so-called second formulation. The effect of the initial rise and the concentrated force on non-linear behavior of the arch was analyzed. It is found that only the symmetrical modes shapes are influenced by the initial rise, these results are due to the fact that when the initial rise is increased, the flexural rigidity of the arch increases. The passage from straight beam to curved beam is very sensitive and depends on the functionality of the future structure, but it is known that curved beam or shallow arch are very performed in case of structure which will support a higher load, because it cans resist more and avoid the earlier deflection of structure. 


\section{References}

[1] Fakhreddine H., Adri A., Chajdi M., Rifai S., Benamar R. A multimode approach to geometrically non-linear forced vibration of beams carrying point masses. Diagnostyka, Vol. 21, Issue 4, 2020, p. 23-33.

[2] Chajdi M., Ahmed A., El Bikri K., Benamar R. Analysis of the associated stress distributions to the nonlinear forced vibrations of functionally graded multi-cracked beams. Diagnostyka, Vol. 22, Issue 1, 2021, p. 101-112.

[3] Ahmed A., Rhali B. Linear and geometrically non-linear frequencies and mode shapes of beams carrying a point mass at various locations. an analytical approach and a parametric study. Diagnostyka, Vol. 18, Issue 2, 2017, p. 13-21.

[4] Benamar R., Bennouna M. M. K., White R. G. The effects of large vibration amplitudes on the mode shapes and natural frequencies of thin elastic structures part I: simply supported and clamped-clamped beams. Journal of Sound and Vibration, Vol. 149, Issue 2, 1991, p. 179-195.

[5] Moussaoui F., Benamar R., White R. G. The effects of large vibration amplitudes on the mode shapes and natural frequencies of thin elastic shells, part I: coupled transverse-circumferential mode shapes of isotropic circular cylindrical shells of infinite length. Journal of Sound and Vibration, Vol. 232, Issue 5, 2000, p. 917-943.

[6] Boutahar L., El Bikri K., Benamar R. A Homogenization procedure for geometrically non-linear free vibration analysis of functionally graded annular plates with porosities, resting on elastic foundations. Ain Shams Engineering Journal, Vol. 7, Issue 1, 2016, p. 313-333.

[7] Bekkaye Merrimi E., EI Bikri K., Benamar R. Geometrically non-linear steady state periodic forced response of a clamped-clamped beam with an edge open crack. Comptes Rendus Mécanique, Vol. 339, Issue 11, 2011, p. 727-742.

[8] Outassafte O., Adri A., Yassine El Khouddar, Rifai S., Benamar R. Geometrically non-linear free in-plane vibration of circular arch elastically restrained against rotation at the two ends. International Journal of Engineering Trends and Technology, Vol. 261, 2021, p. 113515.

[9] Raymond Plaut H., Niels Olhoff Optimal forms of shallow arches with respect to vibration and stability. Journal of Structural Mechanics, Vol. 11, Issue 1, 1982, p. 81-88.

[10] Nayfeh A. H., Kreider W. Investigation of Natural Frequencies and Mode Shapes of Buckled Beams. AIAA Journal, Vol. 33, Issue 6, 1995, p. 1121-1126.

[11] Lacarbonara W., Rega G. Resonant non-linear normal modes. part ii: activation/orthogonality conditions for shallow structural systems. International Journal of Non-Linear Mechanics, Vol. 38, Issue 6, 2003, p. 873-887.

[12] Yi Z., Stanciulescu I. Nonlinear normal modes of a shallow arch with elastic constraints for two-toone internal resonances. Nonlinear Dynamics, Vol. 83, 2016, p. 1577-1600.

[13] Tsiatas G. C., Babouskos N. G. Linear and geometrically nonlinear analysis of non-uniform shallow arches under a central concentrated force. International Journal of Non-Linear Mechanics, Vol. 92, 2017, p. 92-101.

[14] Ye S.-Q., Mao X.-Y., Ding H., Ji J.-C., Chen L.-Q. Nonlinear vibrations of a slightly curved beam with nonlinear boundary conditions. International Journal of Mechanical Sciences, Vol. 168, 2020, p. 105294.

[15] Blair K. B., Krousgrill C. M., Farris T. N. Non-linear dynamic response of shallow arches to harmonic forcing. Journal of Sound and Vibration, Vol. 194, Issue 3, 1996, p. 353-367.

[16] Lakrad F., Schiehlen W. Effects of a low frequency parametric excitation. Chaos, Solitons and Fractals, Vol. 22, Issue 5, 2004, p. 1149-1164.

[17] Ozkaya E., Sarigul M., Boyaci H. Nonlinear transverse vibrations of a slightly curved beam resting on multiple springs. International Journal of Acoustics and Vibration, Vol. 21, Issue 4, 2016, p. $379-391$.

[18] El Kadiri M., Benamar R., White R. G. Improvement of the semi-analytical method, for determining the geometrically non-linear response of thin straight structures. Part I: application to clamped-clamped and simply supported-clamped beams. Journal of Sound and Vibration, Vol. 249, Issue 2, 2002, p. 263-305.

[19] Azrar L., Benamar R., White R. G. A semi-analytical approach to the non-linear dynamic response problem of beams at large vibration amplitudes, part ii: multimode approach to the steady state forced periodic response. Journal of Sound and Vibration, Vol. 255, Issue 1, 2002, p. 1-41. 


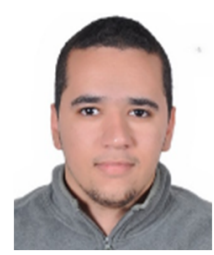

Omar Outassafte Ph.D. student at the Hassan II University of Casablanca, and member of the Mechanics, Manufacturing and Industrial Engineering laboratory. As one of the primary authors of this work; he helped with formulation, programming and writing this paper.

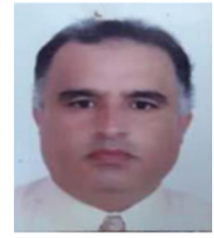

Ahmed Adri is a professor at the Mechanical Engineering Department of ESTC High School of Technology, University Hassan II of Casablanca (Morocco). He obtained his Engineering and Ph.D. in Mechanical Engineering from the EMI and ENSEM Schools of Engineering (Morocco). He is a member of LMPGI Research Laboratory. His fields of research are quality, project and supply chain managements and nonlinear vibration. $\mathrm{He}$ helped with methodology and management activities. He is the leader of this research.

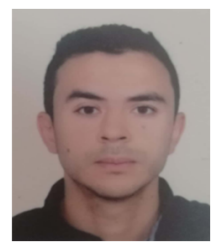

Yassine Elkhouddar is a Ph.D. student at the Hassan II University of Casablanca, and member of the Mechanics, Manufacturing and Industrial Engineering laboratory, he is also a professor of mechanical engineering in in preparatory class for Engineering School, Settat, Morocco. He helped in programming and ideas during this research.

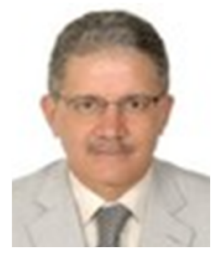

Said Rifai obtained his Ph.D. in industrial acoustics in 1988 from the Poitier University (France). He is a member of the LMPGI Research Laboratory. He is also certified in management and improvement of the quality from the ETS High School of Technology and College Bois de Boulogne Montreal (Canada). He is involved in training engineering and continuing education. His research interests are primarily in the fields of quality, safety and environmental managements and logistics. He helped in management, coordination for the research activity.

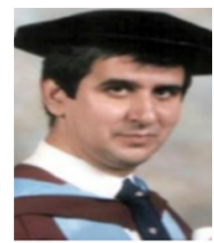

Rhali Benamer is a professor of mechanical engineering and vibrations at the Mohammadia School of Engineering (EMI) - Mohammed V University of Rabat, Morocco. He got his engineering degree in civil engineering in 1982 from the Ecole Nationale des Ponts et Chaussées of Paris, France. He received his Ph.D. in 1990 from the Institute of Sound and Vibration at the University of Southampton, United Kingdom. He helped with methodology. 